\title{
B2.5-Eunomia simulations of Magnum-PSI detachment experiments: II. Collisional processes and their relevance
}

\author{
R. Chandra ${ }^{1}$, H. J. de Blank ${ }^{1}$, P. Diomede ${ }^{2}$, E. Westerhof ${ }^{1}$ \\ ${ }^{1}$ DIFFER - Dutch Institute for Fundamental Energy Research, De Zaale 20, \\ 5612 AJ Eindhoven, The Netherlands \\ ${ }^{2}$ Maastricht University, Minderbroedersberg 4-6, 6211 LK Maastricht, The \\ Netherlands \\ E-mail: r.chandra@differ.nl
}

\begin{abstract}
Detachment is achieved in Magnum-PSI by increasing the neutral background pressure in the target chamber using gas puffing. The plasma is studied using the B2.5 multi fluid plasma code B2.5 coupled with Eunomia, a Monte Carlo solver for neutral species. This study focuses on the effect of increasing neutral background pressure to the plasma volumetric loss of particle, momentum and energy. The plasma particle and energy loss almost linearly scale with the increase of neutral background pressure, while the momentum loss does not scale as strongly. Plasma recombination processes include molecular activated recombination (MAR), dissociative attachment, and atomic recombination. Atomic recombination, which includes radiative and three-body recombination, is the most relevant plasma process in reducing the particle flux and, consequently, the heat flux to the target. The low temperature where atomic recombination becomes dominant is achieved by plasma cooling via elastic $\mathrm{H}^{+}-\mathrm{H}_{2}$ collisions. The transport of vibrationally excited $\mathrm{H}_{2}$ molecules out of the plasma serves as an additional electron cooling channel with relatively small contribution. Additionally, the transport of highly vibrational $\mathrm{H}_{2}$ has a significant impact in reducing the effective MAR and dissociative attachment collision rates and should be considered properly. The relevancy of MAR and atomic recombination occupy separate electron temperature regimes, respectively, at $T_{\mathrm{e}}=1.5 \mathrm{eV}$ and $T_{\mathrm{e}}=0.3 \mathrm{eV}$, with dissociative attachment being relevant in the intermediary. Plasma cooling via elastic $\mathrm{H}^{+}-\mathrm{H}_{2}$ collisions is effective at $T_{\mathrm{e}} \leq 1$ $\mathrm{eV}$.

Keywords:linear plasma device, detachment, fluid-kinetic code, plasma-neutral interaction
\end{abstract}




\section{Introduction}

The discovery of plasma detachment in the past 1 has led to extensive research in understanding the mechanism that may prevent the destruction of plasma facing materials in future tokamak reactors. Several recent reviews of detachment studies in tokamak 2. 3] and linear plasma devices [4] highlighted the importance of volumetric losses in reducing the particle and heat flux in the divertor plasma region. One of them is the process of plasma recombination, which effectively transports the plasma particles and energy through the recombined neutral particles that are not confined by the magnetic field. Thus, the energy will be deposited in a larger surface area. Plasma recombination is achieved via radiative and threebody recombination at low temperatures $(<1 \mathrm{eV})$ and mediated via the molecular activated recombination (MAR) process at higher temperatures $(>1 \mathrm{eV})$. For either of these two processes to be relevant, a significant drop of plasma temperature must occur before the plasma reaches the target material. This drop is driven by the loss of plasma parallel momentum and energy through plasma-neutral interaction, via charge exchange, elastic collision (atomic or molecular) or radiative processes. Numerical codes are often used to investigate the contribution of these three main processes, recombination, momentum, and radiative losses [5 11], while at the same time rely on the correct inclusion of such processes in the code. A validation between such codes and experimental measurement in linear plasma devices can provide more insight into the actual processes involved in the more complex tokamak plasmas, given that the relevant plasma parameters can be achieved. The detailed modelling of processes involved in plasma detachment is rather limited in linear plasma devices. A recent modelling study using the LINDA code in GAMMA10/PDX [12 showed a reduction in electron temperature due to injection of $\mathrm{H}$ neutrals. However, processes involving hydrogen molecules such as molecular activated recombination (MAR) was not yet included. Another study used SOLEDGE2D-EIRENE and comparison with experimental data to investigate plasma detachment in Pilot-PSI [13, highlighting the importance of ion-molecule elastic collision in determining the ion flux reaching the target. However, the simulation overestimated the measured electron temperature and the absence of vibrational excitation of the hydrogen molecules were suspected to be the cause.

Investigations into detachment properties were recently conducted 14] using the linear plasma device Magnum-PSI 15]. Magnum-PSI produces plasma beams with temperatures below $5 \mathrm{eV}$, similar to tokamak plasma conditions in front of the target in (partially) detached divertor regime. Detachment in Magnum-PSI is achieved by increasing the neutral gas pressure in the target chamber. $\mathrm{H}-\alpha$ emission from the plasma is very localized at the target in the case of very low neutral gas pressures. As the neutral pressure increases, more of the plasma volume is emitting light, and eventually the volume is visibly moving away from the target. The plasma parameters observed using Thomson scattering (TS) during this transition have been simulated using the coupled plasma and neutral particle code B2.5 [16]-Eunomia [17] and reported in [18]. B2.5-Eunomia includes molecular hydrogen processes in vibrationally excited states and treats vibrationally excited $\mathrm{H}_{2}$ molecules as test particles. The increase of neutral gas pressure in the target chamber is achieved by gas puffing. The simulation parameters are adjusted such that the resulting plasma parameters are matched as close as possible with Thomson scattering measurements when there is no gas puffing. Gas puffing is then introduced in the simulation to increase the neutral pressure in the target chamber, while keeping all other simulation parameters unchanged. The electron temperature and density in the target chamber are compared to TS measurements in the same location and gas pressure value. Quantitative agreements are found in the comparisons of electron temperatures, which are the sensitive parameter that dictates the collision processes between the plasma and neutral gas 18. The increase of gas pressure in the target chamber has been observed to reduce the heat and particle flux reaching the tungsten disc target, and this behaviour was qualitatively replicated in the simulation.

This paper is a companion paper of [18], together giving a description and analysis of detachment experiments in Magnum-PSI. While [18] described the detachment experiments and simulations in terms of plasma and gas parameters, the present paper focuses on the collisional processes that cause both the change of $\mathrm{H}-\alpha$ emission volume and the reduction of target fluxes in the transition to a detached state. This paper is structured as follows: first, an overview of the code B2.5-Eunomia is discussed in section 2, The 
global particle, momentum and energy balance of the simulation results are discussed in section 3. The detailed discussion of collisional processes affecting detachment characteristics is in section 4. The effect of vibrational hydrogen molecule transport to the plasma particle balance is discussed in section 5. And finally, the relevance of collisional processes in different plasma regimes is discussed in section 6. The conclusion and outlook of this paper are presented in section 7 .

\section{B2.5-Eunomia}

\subsection{Description}

A brief description of B2.5-Eunomia will be provided here to help elucidate the results presented in this paper. For a more detailed explanation of the code, we recommend reading the cited sources for each code. B2.5 $[16$ is a multi-fluid plasma code that solves the conservation equations of particle, momentum and energy derived by Braginskii [19] in two spatial dimensions. These equations, in steady state, can be written as follows [16]:

$$
\begin{aligned}
& \nabla \cdot\left(n_{\mathrm{i}} \boldsymbol{V}_{\mathrm{i}}\right)=S_{\mathrm{i}} \\
& \nabla \cdot\left(n_{\mathrm{e}} \boldsymbol{V}_{\mathrm{e}}\right)=S_{\mathrm{e}} \\
& \nabla \cdot\left(m_{\mathrm{i}} n_{\mathrm{i}} \boldsymbol{V}_{\mathrm{i}} \boldsymbol{V}_{\mathrm{i}}\right)=-\nabla p_{\mathrm{i}}-\nabla \cdot \Pi_{\mathrm{i}} \\
& +Z_{\mathrm{i}} e n_{\mathrm{i}}\left(\boldsymbol{E}+\boldsymbol{V}_{\mathrm{i}} \times \boldsymbol{B}\right)+\boldsymbol{R}+\boldsymbol{S}_{m_{\mathrm{i}} \boldsymbol{V}_{\mathrm{i}}} \\
& -\nabla p_{\mathrm{e}}-e n_{\mathrm{e}}\left(\boldsymbol{E}+\boldsymbol{V}_{\mathrm{e}} \times \boldsymbol{B}\right)-\boldsymbol{R}=0 \\
& \nabla \cdot\left[\left(\frac{5}{2} n_{\mathrm{i}} T_{\mathrm{i}}+\frac{m_{\mathrm{i}} n_{\mathrm{i}}}{2}\left|\boldsymbol{V}_{\mathrm{i}}\right|^{2}\right) \boldsymbol{V}_{\mathrm{i}}+\boldsymbol{q}_{\mathrm{i}}+\Pi_{\mathrm{i}} \cdot \boldsymbol{V}_{\mathrm{i}}\right]= \\
& +\left(Z_{\mathrm{i}} e n_{\mathrm{i}} \boldsymbol{E}-\boldsymbol{R}\right) \cdot \boldsymbol{V}_{\mathrm{i}}-Q_{\mathrm{ei}}+S_{E_{\mathrm{i}}} \\
& \nabla \cdot\left[\frac{5}{2} n_{\mathrm{e}} T_{\mathrm{e}} \boldsymbol{V}_{\mathrm{i}}+\boldsymbol{q}_{\mathrm{e}}\right]=-e n_{\mathrm{e}} \boldsymbol{E} \cdot \boldsymbol{V}_{\mathrm{e}}+\boldsymbol{R} \cdot \boldsymbol{V}_{\mathrm{i}} \\
& +Q_{\mathrm{ei}}+S_{E_{\mathrm{e}}}
\end{aligned}
$$

where (1) is the particle balance for ions, (2) is the particle balance for electrons, (3) is the ion momentum balance, (4) is the generalized Ohm's law, (5) and (6) are, respectively, the energy balance for ions and electrons. The B2.5 simulation grid is defined using the cylindrical coordinate system with axisymmetry in the $\phi$ direction. The magnetic field is parallel to the $\mathrm{Z}$ direction and perpendicular to the $\mathrm{R}$ direction. We can define the positive direction of plasma flow, $\boldsymbol{V}_{\mathrm{i}}$, as going from upstream to downstream in the $\mathrm{Z}$ direction, and as going from the center of the plasma beam to the edge in the $\mathrm{R}$ direction.

Eunomia 17 is a non-linear Monte Carlo code for neutral particles that solves the equilibrium state of the neutrals as well as source terms for fluid plasma calculations. Neutral test-particles are emitted from known neutral sources and during their trajectories a collision partner is drawn from a local shifted Maxwellian plasma background accounting the plasma flow velocity supplied by B2.5. The averaged accumulated collisions with this partner will result in the source terms in (1) - (6), specifically the $S_{\mathrm{i}}, S_{\mathrm{e}}, \boldsymbol{S}_{m_{\mathrm{i}} \boldsymbol{V}_{\mathrm{i}}}, S_{E_{\mathrm{i}}}$ and $S_{E_{\mathrm{e}}}$, that is supplied back to B2.5 for calculations of the next time step. This coupling process is repeated until equilibrium is reached. For the modelling of Magnum-PSI detachment experiments 20, 21, the list of collisions that are implemented for the results of this paper is presented in table 1 .

\begin{tabular}{|c|c|c|}
\hline Nr. & Collision formula & Database ref. \\
\hline 1 & $\mathrm{H}+e^{-} \rightarrow \mathrm{H}^{+}+2 e^{-}$ & $22 \quad 2.1 .5$ \\
\hline 2 & $\mathrm{H}+e^{-} \rightarrow \mathrm{H}^{*}(n=2)+e^{-}$ & 22.1 .1 \\
\hline 3 & $\mathrm{H}+\mathrm{H}^{+} \rightarrow \mathrm{H}^{+}+\mathrm{H}$ & 22 \\
\hline 4 & $\mathrm{H}+\mathrm{H} \rightarrow$ elastic & 25 \\
\hline 5 & $\mathrm{H}+\mathrm{H}_{2} \rightarrow$ elastic & 25 \\
\hline 6 & $\mathrm{H}_{2}+\mathrm{H}_{2} \rightarrow$ elastic & 25 \\
\hline 7 & $\mathrm{H}+\mathrm{H}^{+} \rightarrow$ elastic & $230.1 \mathrm{~T}$ \\
\hline 8 & $\mathrm{H}_{2}+\mathrm{H}^{+} \rightarrow$ elastic & $230.3 \mathrm{~T}$ \\
\hline 9 & $\mathrm{H}^{+}+e^{-} \rightarrow \mathrm{H}$ & 232.1 .8 \\
\hline 10 & $\mathrm{H}^{+}+\mathrm{H}_{2}(v=0-14) \rightarrow \mathrm{H}+\mathrm{H}_{2}^{+}$ & $24 \quad 2.12$ \\
\hline 11 & $\mathrm{H}_{2}^{+}+e^{-} \rightarrow \mathrm{H}+\mathrm{H}^{*}$ & Spontaneous \\
\hline 12 & $\mathrm{H}_{2}^{2}(v=i)+e^{-} \rightarrow \mathrm{H}_{2}(v=i+1)+e^{-}$ & $242 . \mathrm{iv}(\mathrm{i}+1)$ \\
\hline 13 & $\mathrm{H}_{2}(v=i)+e^{-} \rightarrow \mathrm{H}_{2}(v=i-1)+e^{-}$ & $242 . i v(\mathrm{i}-1)$ \\
\hline 14 & $\mathrm{H}_{2}(v=0-14)+e^{-} \rightarrow \mathrm{H}+\mathrm{H}^{-}$ & 24.13 \\
\hline 15 & $\mathrm{H}^{-}+\mathrm{H}^{+} \rightarrow \mathrm{H}+\mathrm{H}^{*}$ & Spontaneous \\
\hline 16 & $\mathrm{H}_{2}(v=0-14)+e^{-} \rightarrow \mathrm{H}+\mathrm{H}+e^{-}$ & 242.11 \\
\hline
\end{tabular}

Table 1: Collision lists used in the Eunomia code. The databases used are HYDHEL 22], AMJUEL 23], and H2VIBR [24, with the respective reaction numbers stated. For collisions between neutral particles the cross-section are calculated using the Lennard-Jones potential of the atom or molecule 25]. Spontaneous means the process occurs simultaneously when the ionic reactant is produced by another process.

\subsection{Simulations of Magnum-PSI detachment experiments}

The detached plasma condition was experimentally investigated in Magnum-PSI by locally increasing the neutral gas pressure near the target 14,20. The experiments are done using two different plasma source settings. When the electron density and temperature profiles are measured using TS near the target location, their peak values correspond to two different plasma regimes. The first experiment, where $n_{\mathrm{e}}=10^{20} \mathrm{~m}^{-3}$ and $T_{\mathrm{e}}=4 \mathrm{eV}$, is labeled the low density case. The second experiment, where $n_{\mathrm{e}}=5 \cdot 10^{20} \mathrm{~m}^{-3}$ and $T_{\mathrm{e}}=1$ $\mathrm{eV}$, is labeled the high density case. For both of these experiments, while the source settings remained fixed, gas puffing is used in the target chamber to increase the local neutral pressure, $p_{\mathrm{n}}$. The electron 
temperature near the target is observed to decline with increasing neutral pressure. There is also evidence of reduction in heat flux to the target with increasing $p_{\mathrm{n}}$ measured with calorimetry. Using B2.5-Eunomia, the plasma profiles in both experiments during zero gas puffing are replicated using the limited information available from the experiment to constrain the input parameters of B2.5 and Eunomia. Input parameters with no information from the experiment are used as adjustment parameters to match the $n_{\mathrm{e}}$ and $T_{\mathrm{e}}$ profiles that are measured using TS at the same coordinates. When the matches are achieved, all input parameters are fixed and gas puffing are introduced in the target chamber domain, thereby increasing the neutral pressure as in the experiments. Therefore, B2.5-Eunomia can produce the plasma and neutral distribution for different neutral pressures within the target chamber. The details of the methodology and comparison with experimental measurements can be found in the first part of this study [18]. In this paper, as the second part of this study, the plasma solution will be further analyzed to understand the effects of increasing the local neutral pressure to the observed plasma parameters and heat flux to the target. Specifically, the role of different collision processes between the plasma and neutrals will be discussed in detail.

\subsection{Direct sampling of ion-neutral collisions in Eunomia}

As explained in section 2.1. Eunomia launches testparticles to interact with the background Maxwellian plasma obtained from B2.5 in order to calculate the plasma source terms caused by interaction with neutral particles. The contributions of the collision events in each grid cell can be described as follows 17:

$$
\begin{aligned}
S_{\mathrm{i}} & =\Gamma_{\text {col }} \Delta P_{\mathrm{i}} / \Delta V \\
S_{\mathrm{e}} & =\Gamma_{\text {col }} \Delta P_{\mathrm{e}} / \Delta V \\
S_{m_{\mathrm{i}} \boldsymbol{V}_{\mathrm{i}}} & =\Gamma_{\text {col }} m(\boldsymbol{v}-\boldsymbol{u}) / \Delta V \\
S_{E_{\mathrm{i}}} & =\Gamma_{\text {col }} \frac{1}{2} m\left(\left|\boldsymbol{v}-\boldsymbol{V}_{\mathrm{i}}\right|^{2}-\left|\boldsymbol{u}-\boldsymbol{V}_{\mathrm{i}}\right|^{2}\right) / \Delta V \\
S_{E_{\mathrm{e}}} & =\Gamma_{\text {col }} \Delta E_{\mathrm{i}} / \Delta V
\end{aligned}
$$

here $\Delta P_{\mathrm{i}}$ and $\Delta P_{\mathrm{e}}=-1,0$, or 1 depending on the collision process, $\boldsymbol{u}$ and $\boldsymbol{v}$ are the velocity vectors of the particle before and after the collision event, $\Delta E_{\mathrm{i}}$ is the electron energy gain or loss, and $\Delta V$ is the volume of the grid cell. $\Gamma_{\text {col }}$ is the number of real collision rate in the cell. $\Gamma_{\mathrm{col}}$ is approximated from different neutral sources that are specified in the simulation. $\Gamma_{\mathrm{col}}$ is approximated by $\Gamma_{\mathrm{col}}=\Gamma_{\mathrm{tp}, \mathrm{col}} \Gamma_{\mathrm{rp}} / N_{\mathrm{tp}}$, where $\Gamma_{t p, c o l}$ is the number of test-particle collision events, $\Gamma_{\mathrm{rp}}$ is the real neutral flux from the neutral source (for example gas puffing), and $N_{\text {tp }}$ is the total number of test-particles launched from the neutral source. When running B2.5-Eunomia to obtain a converged solution, the neutral source contributions are summed for all collision events thereby providing a net source term in each cell. To separate the contributions of each collision event a second simulation with Eunomia is performed. The steady-state plasma solution from the first simulation is used as the plasma background and test-particles are launched again from the same neutral sources. However, for the second simulation the B2.5 continuity equations are not solved. This is achieved by specifying a very small time step value in the B2.5 code $\left(10^{-20} \mathrm{~s}\right)$, and so the plasma is frozen in place. B2.5-Eunomia is run for a few cycles, and for each cycle the data of (7) are outputted, which contains the value of these terms for each unique collision events in every cell.

\section{Global particle, momentum and power balance}

In this paper, the analysis will be limited to the simulation domain of the Magnum-PSI target chamber. From [18], it was shown that the change in neutral pressure is localized to the target chamber only. The upstream location is defined as the entrance to the target chamber, the downstream location is the target, and the radial boundary is the radial outer edge of the plasma beam as illustrated in figure 1. The integral

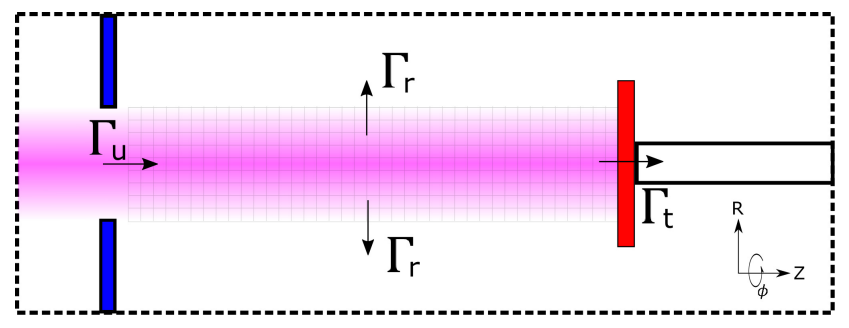

Figure 1: A simplified picture of the plasma beam in the target chamber. The plasma enters the chamber from the skimmer (blue) and ends at the target (red). The upstream flux $\Gamma_{\mathrm{u}}$ is defined in the skimmer entrance and the downstream flux $\Gamma_{\mathrm{t}}$ is at the target. The radial flux $\Gamma_{\mathrm{r}}$ is the total particle flux leaving the plasma beam radially. The arrows indicate the flow direction. In this illustration the chamber walls lie outside the bounding box.

form of (1) - (6) can be written as:

$$
\oint_{A} \boldsymbol{\Gamma} \cdot \mathbf{d} \mathbf{A}=\int_{V} S d V
$$

In B2.5 the quantity $\boldsymbol{\Gamma}$ is defined on cell boundaries, while $S$ is defined in the cell volume. We define $\Gamma=\boldsymbol{\Gamma} \cdot \hat{\mathrm{n}}$ where $\hat{\mathrm{n}}$ is the vector normal to the surface $A$ pointing 
outward of the volume. From code output, the particle balance in 12 is written as:

$\sum_{\mathrm{u}} \Gamma_{\mathrm{u}} A_{\mathrm{u}}+\sum_{\mathrm{t}} \Gamma_{\mathrm{t}} A_{\mathrm{t}}+\sum_{\mathrm{r}} \Gamma_{\mathrm{r}} A_{\mathrm{r}}=\sum_{c} S_{\mathrm{i}, \mathrm{c}} V_{c}$

where the subscript $\mathrm{u}, \mathrm{t}$ and $\mathrm{r}$ denote the upstream, downstream, and radial location of the bounding surface, vol is the volumetric source due to collisions, $A$ is the surface area, $V$ is the cell volume, and $c$ indicates the cell number. We treat the individual terms $\sum \Gamma A$ as source terms and noting in figure 1 that $\boldsymbol{\Gamma}_{\mathrm{u}}$ is antiparallel to $\hat{n}, 13$ simplifies to:

$\Phi_{\mathrm{u}}=\Phi_{\mathrm{t}}+\Phi_{\mathrm{r}}+\Phi_{\mathrm{vol}}$.

where $\Phi_{\mathrm{vol}}$ is the total particle sink so that $\Phi_{\mathrm{vol}}=$ $-\sum_{c} S_{\mathrm{i}, \mathrm{c}} V_{c}$. The steady state particle balance is shown in figure 2 for all cases. It is readily shown that (14)

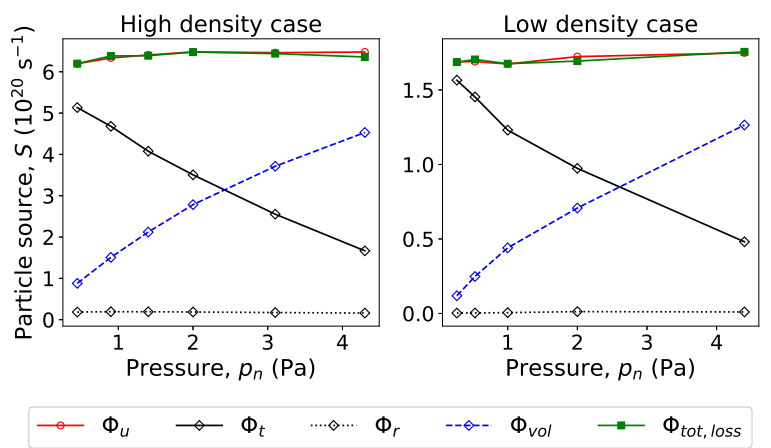

Figure 2: The total plasma particle balance in the plasma beam. The incoming particle flux, $\Phi_{\mathrm{u}}$ must equal the total particle loss, $\Phi_{\text {tot,loss }}$ in steady-state which is shown here. $\Phi_{\text {tot,loss }}=\Phi_{\mathrm{vol}}+\Phi_{\mathrm{r}}+\Phi_{\mathrm{t}}$, where $\Phi_{\mathrm{t}}$ is the particle flux reaching the target, $\Phi_{\mathrm{vol}}$ is the total particle sink, and $\Phi_{\mathrm{r}}$ is the particle flux perpendicular to the magnetic field. $\Phi_{\mathrm{t}}$ is reduced due to increasing $\Phi_{\text {vol }}$ with increasing neutral background pressure, $p_{n}$

is satisfied with the slight visible differences due to the numerical tolerance in B2.5's solution of the plasma equations. It is also shown here that $\Phi_{\mathrm{t}}$ reduces with increasing $p_{\mathrm{n}}$, as discussed in 18. However, it is shown now that $\Phi_{\mathrm{vol}} \propto p_{\mathrm{n}}$ which reflects the expectation that increasing the neutral background pressure will increase the rate of plasma-neutral interaction.

The same approach used for 12 and 13 can be applied to the ion momentum balance equation, (3) resulting in:

$\sum_{\mathrm{u}} \Gamma_{\mathrm{u}} m_{\mathrm{i}} \boldsymbol{V}_{i, u} A_{\mathrm{u}}+\sum_{\mathrm{t}} \Gamma_{\mathrm{t}} m_{\mathrm{i}} \boldsymbol{V}_{i, t} A_{\mathrm{t}}+\sum_{\mathrm{r}} \Gamma_{\mathrm{r}} m_{\mathrm{i}} \boldsymbol{V}_{i, r}$

$=\sum_{c}\left(S_{m_{\mathrm{i}} \boldsymbol{V}_{\mathrm{i}}, c}+S_{\nabla p, c}\right) V_{c}$

to yield the simple form of momentum flux balance:

$F_{\mathrm{u}}=-F_{p}+F_{\mathrm{t}}+F_{\mathrm{r}}+F_{\mathrm{vol}}$ where $F_{\text {vol }}$ is the total momentum sink so that $F_{\text {vol }}=-\sum_{c} S_{m_{\mathrm{i}} V_{\mathrm{i}}, c} V_{c}, \quad F_{p}=\sum_{c} S_{\nabla p, c} V_{c}$ is the momentum source due to pressure gradient along the flow direction, the parallel viscosity term in (3) is combined in the flux term on the left hand side, and the $V \times B$ term vanishes because of their parallel direction. The steady-state momentum balance is shown in figure 3 and the slight differences that are visible correspond to numerical tolerance. Two

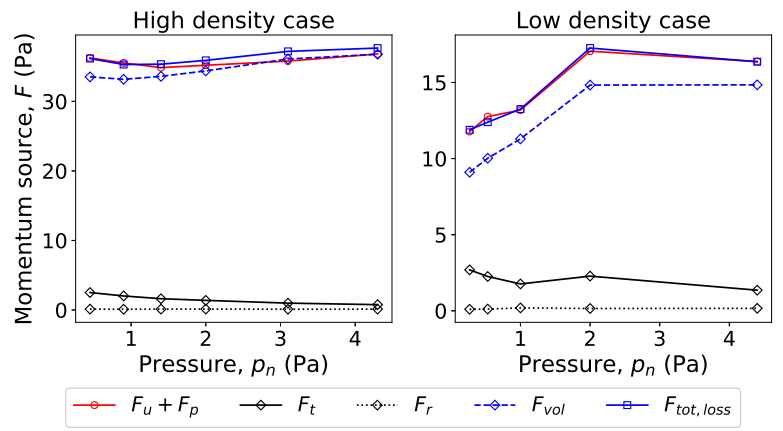

Figure 3: The plasma momentum balance in the plasma beam, normalized to the target cross-section area to yield the pressure unit Pa. The momentum balance is $F_{\mathrm{u}}+F_{p}=F_{\text {tot,loss }}$, where $F_{\mathrm{u}}$ is the momentum flux entering the target chamber and $F_{p}$ is the pressure gradient term. $F_{\text {tot,loss }}=F_{\mathrm{t}}+F_{\mathrm{vol}}+F_{\mathrm{r}}$, where $F_{\mathrm{t}}$ is the momentum flux to the target, $F_{\mathrm{vol}}$ is the volumetric momentum loss and $F_{\mathrm{r}}$ is the radial momentum flux.

distinct characteristics are observed for the momentum balance for both cases. First, significant volumetric momentum loss can already be seen when no gas puffing has yet been applied. Second, the dependence of the volumetric momentum losses on the neutral gas pressure is rather weak. A clearer picture of this characteristics will be shown later in section 4.2

For the heat balance, (5) and (6) are combined to gain the total heat balance. Using the form in 12 , the heat balance is written as:

$\sum_{\mathrm{u}} q_{\mathrm{u}}^{\prime} A_{\mathrm{u}}+\sum_{\mathrm{t}} q_{\mathrm{t}}^{\prime} A_{\mathrm{t}}+\sum_{\mathrm{r}} q_{\mathrm{r}}^{\prime} A_{\mathrm{r}}$
$=\sum_{c}\left(S_{E_{\mathrm{i}}, c}+S_{E_{\mathrm{e}}, c}+S_{J, c}\right) V_{c}$

where $q^{\prime}$ is the flux terms in the square brackets in (5) and (6) combined, and $S_{\mathrm{J}}$ is the heat source term due to Joule heating. The simple heat balance is written in the form:

$Q_{\mathrm{u}}^{\prime}=-Q_{\mathrm{J}}+Q_{\mathrm{t}}^{\prime}+Q_{\mathrm{r}}^{\prime}+Q_{\mathrm{vol}}^{\prime}$

where $Q_{\mathrm{vol}}^{\prime}$ is the total heat sink so that $Q_{\mathrm{vol}}^{\prime}=$ $-\sum_{c}\left(S_{E_{\mathrm{i}}, c}+S_{E_{\mathrm{e}}, c}\right) V_{c}$ and $Q_{\mathrm{J}}=\sum_{c} S_{J, c} V_{c}$. The heat flux reaching the target consist of conduction, 
convection, and the energy deposited from the ionization potential of ions. The ionization potential flux is simply the particle flux times the potential, $\Phi_{\mathrm{t}} E_{\alpha}$, where $E_{\alpha}=-13.6 \mathrm{eV}$. This flux can be included in 18 , such that $Q_{\mathrm{t}}$ reflects the energy deposited to the target, and can be modified into:

$\left(Q_{\mathrm{u}}^{\prime}+\Phi_{\mathrm{u}} E_{\alpha}\right)+Q_{\mathrm{J}}=\left(Q_{\mathrm{t}}^{\prime}+\Phi_{\mathrm{t}} E_{\alpha}\right)$

$+\left(Q_{\mathrm{r}}^{\prime}+\Phi_{\mathrm{r}} E_{\alpha}\right)+\left(Q_{\mathrm{vol}}^{\prime}+\Phi_{\mathrm{vol}} E_{\alpha}\right)$

where $\Phi_{\mathrm{u}}, \Phi_{\mathrm{t}}, \Phi_{\mathrm{r}}$ and $\Phi_{\mathrm{vol}}$ are the terms defined in the overall particle balance (14), and $Q_{\mathrm{J}}$ is moved to the left hand side. In figure 4 , the energy balance of 19 is demonstrated with slight differences due to numerical tolerance in the B2.5 plasma solution. Ohmic heating

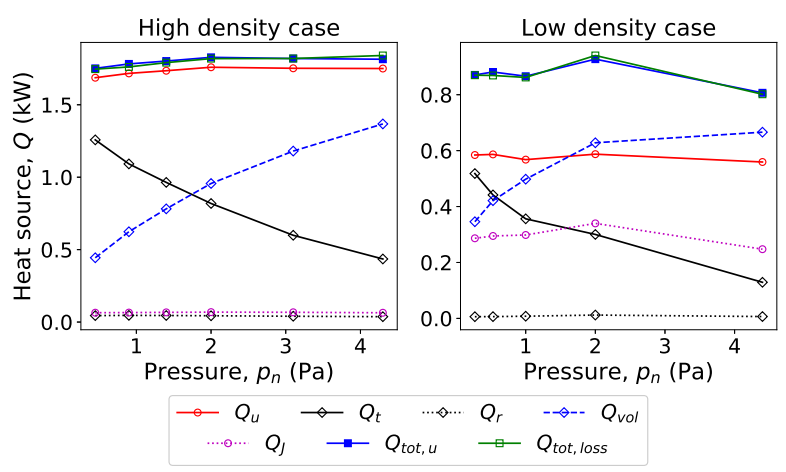

Figure 4: The total plasma energy balance in the plasma beam, summed for both electron and ions. The incoming energy flux, $Q_{t o t, u}$ must equal the total energy loss, $Q_{\text {tot,loss }}$ in steady-state which is shown here. $Q_{t o t, u}=Q_{\mathrm{u}}+Q_{J}$, where $Q_{\mathrm{u}}$ is the heat flux entering the target chamber, and $Q_{J}$ is the total Ohmic heating. $Q_{\mathrm{tot}, \text { loss }}=Q_{\mathrm{t}}+Q_{\mathrm{vol}}+Q_{\mathrm{r}}$, where $Q_{\mathrm{t}}$ is the heat flux reaching the target, $Q_{\mathrm{vol}}$ is the total heat sink, and $Q_{\mathrm{r}}$ is the heat flux perpendicular to the magnetic field. The $\mathrm{u}, \mathrm{t}, \mathrm{r}$, and vol terms include the ionization potential flux, such that $Q=Q^{\prime}+\Phi E_{\alpha}$ as described in 19. $Q_{\mathrm{t}}$ is reduced due to increasing $Q_{\mathrm{vol}}$ with increasing neutral background pressure, $p_{n}$.

is seen to be a significant heat source term in the low density case, but rather negligible in the high density case. For both cases, this contribution remains constant with increasing $p_{\mathrm{n}}$. Here it is shown that $Q_{\text {vol }} \propto p_{\mathrm{n}}$, similar to the volumetric recombination loss $\Phi_{\mathrm{vol}}$, which is not surprising considering the loss of ionization potential energy through recombination, $\Phi_{\mathrm{vol}} E_{\alpha}$, is rather significant.

It is important to note that heavy impurities are not simulated in this study. In a tokamak, impurities can play a significant role in the plasma energy balance during plasma detachment. Impurity radiation reduces the available power in the SOL for plasma recycling 26]. Impurity species such as nitrogen or argon are seeded intentionally to enhance radiation and accommodate partial plasma detachment 27. As Magnum-PSI already produces plasmas similar to (partially) detached divertor regime, no impurities are necessary to achieve the aforementioned effects. Furthermore, sputtered impurities originating from the target (made from tungsten) and source is negligible in the plasma temperature ranges considered here. Hence, impurity radiation is absent in the energy balance.

\section{Collisional processes responsible for detachment in Magnum-PSI}

The volumetric loss term of particles, momentum and energy, $\Phi_{\text {vol }}, F_{\text {vols }}$ and $Q_{\text {vol }}$ can be decomposed to show the contributions of individual collisional processes, using the data gathered with the sampling method described in section 2.3 .

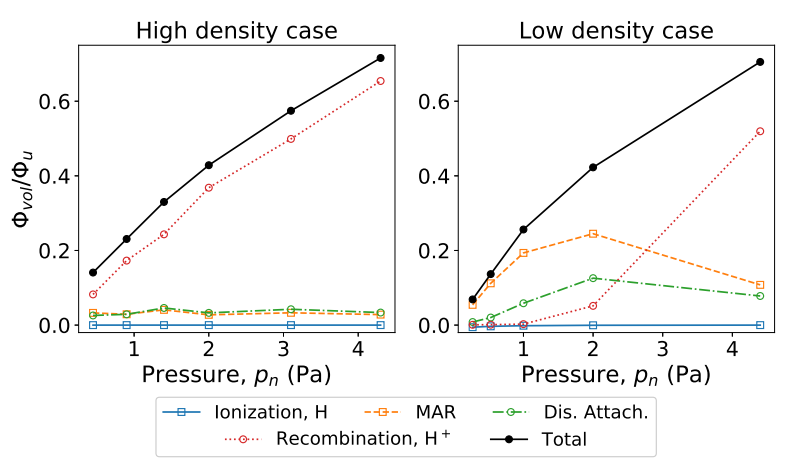

Figure 5: Individual contributions of different plasma collision processes to the volumetric particle loss, $\Phi_{\mathrm{vol}}$, normalized to $\Phi_{\mathrm{u}}$. For the high density case, electronproton recombination is the major particle sink term in all values of, $p_{\mathrm{n}}$. For the low density case, MAR is the dominant sink term for low $p_{\mathrm{n}}$, and a transition occurs at high $p_{\mathrm{n}}$ in which electron-proton recombination is the dominant process.

\subsection{Volume particle loss}

Plasma particles are generated via the ionization of atomic hydrogen (collision 1 in table 1) and removed via the recombination processes: MAR (collision 10 and 11 in table 1), dissociative attachment (collision 14 and 15 in table 1), and electron-proton recombination (collision 9 in table 1 ). The volumetric particle losses for each contributing collision are integrated over the entire plasma beam and shown in figure 5 . There is a significant difference between the two cases, particularly in the role of electron-proton recombination. In the high density case, recombination completely dominates $\Phi_{\mathrm{vol}}$ for all neutral gas pressures $p_{\mathrm{n}}$, and so the relationship $\Phi_{\mathrm{vol}} \propto p_{\mathrm{n}}$ is mainly caused 
by an increase of electron-proton recombination rate. In the low density case, it is only relevant at high $p_{\mathrm{n}}$, where $p_{\mathrm{n}} \geq 2 \mathrm{~Pa}$. Moreover, there is a reversal in terms of the dominant particle loss mechanism at $p_{\mathrm{n}} \leq 2 \mathrm{~Pa}$, where the molecular processes, MAR and dissociative attachment, are dominant. To give a clearer picture of the reversal, the location where these collisions are relevant within the plasma beam are shown in figure 6. The collisions are normalized to the sum of the contributions listed in figure 5 Each row represents a different $p_{\mathrm{n}}$ value: $0.27 \mathrm{~Pa}$ (top row), $2.0 \mathrm{~Pa}$ (middle row) and $4.4 \mathrm{~Pa}$ (bottom row). Red represents where the collision is the most dominant compared to others. During the attached condition (at $0.27 \mathrm{~Pa}$ ), MAR is prevalent in most of the volume. The exception lies at the center of the beam and near the target, where instead of particle loss, ionization of atomic hydrogen contributes as a particle source. As $p_{\mathrm{n}}$ increases, electron-proton recombination starts to take over from the plasma periphery, until it is completely dominating the region closer to the target boundary, and MAR dominance is retracted towards the target chamber entrance. Dissociative attachment seems to never be dominant in all pressures, with some significant rate occurring at intermediary regions between MAR and electron-proton recombination. Ionization is not relevant in both the high and low density case, as shown in figure 5 , and in figure 6 most ionization occurs at the center of the plasma beam where $T_{\mathrm{e}}$ is highest, and near the target where a large atomic hydrogen density region is located due to plasma recombination at the target.

\subsection{Volume momentum loss}

The plasma parallel momentum can be removed via collisions with neutrals through these processes: elastic collisions (collision 7 and 8 in table 1), charge exchange (collision 3 and 10 in table 1), and recombination (collision 9 and 15 in table 11). The total parallel momentum loss via collisions with neutrals is shown in figure 7. The most prominent collisions responsible for momentum loss are $\mathrm{H}^{+}-\mathrm{H}$ charge exchange (CX) and $\mathrm{H}^{+}-\mathrm{H}_{2}$ elastic collision (respectively, collision 3 and 8 in table 1). CX is dominant in the high and low density case at lower neutral pressures $\left(p_{\mathrm{n}} \leq 2.0 \mathrm{~Pa}\right)$ whereas $\mathrm{H}_{2}-\mathrm{H}^{+}$elastic collisions prevails over $\mathrm{CX}$ at higher neutral pressures $\left(p_{\mathrm{n}} \geq 2.0 \mathrm{~Pa}\right)$. The increase of momentum loss through $\mathrm{H}_{2}-\mathrm{H}^{+}$elastic collisions with $p_{\mathrm{n}}$ is expected since the density of $\mathrm{H}_{2}$ is increased by gas puffing. However, the contribution of CX decreases with increasing $p_{\mathrm{n}}$. This can be explained by looking at where $\mathrm{CX}$ is relevant for the low density case in figure 8 The bulk of CX losses occurs in front of the target, where the density of $\mathrm{H}$ is high due to plasma recombination at the target surface.
As the neutral gas pressure increases, more of the plasma momentum is reduced due to elastic $\mathrm{H}_{2}-\mathrm{H}^{+}$ collisions before the plasma reaches the region where $\mathrm{CX}$ is dominant, hence the reduction by $\mathrm{CX}$ is less intense. Additionally, as $p_{\mathrm{n}}$ increases, the particle flux that reaches the target, $\Phi_{\mathrm{t}}$, which acts as the source of plasma surface recombination, decreases. This reduces the local $\mathrm{H}$ density near the target and subsequently the rate of CX collisions (see Appendix B). The weak correlation between $F_{\mathrm{vol}}$ and $p_{\mathrm{n}}$ shown in figure 3 is explained here by the role reversal between $\mathrm{CX}$ and elastic collisions of $\mathrm{H}_{2}-\mathrm{H}^{+}$. The effects of increasing $p_{n}$ effectively replaces the cushioning of recombined atoms with the molecular type. Figure 7 also reveals that elastic collisions of $\mathrm{H}-\mathrm{H}^{+}$are a much smaller momentum loss process compared to CX. In B2.5Eunomia, it is assumed that the scattering angle of ionneutral collisions are anisotropic 28. Elastic collisions of $\mathrm{H}-\mathrm{H}^{+}$mainly result in small angle collisions, and so the momentum transfer of the ion flow is smaller compared to CX.

\subsection{Volume energy loss}

The energy from the plasma can be removed from collisions with ions or electrons. In this paper, ionic energy losses include atomic charge exchange (CX, collision 3 in table 1), elastic $\mathrm{H}-\mathrm{H}^{+}$and $\mathrm{H}_{2}-\mathrm{H}^{+}$ collision (nr. 7 and 8 in table 1). Electronic processes include the particle loss processes mentioned in section 4.1 and in addition: excitation of atomic hydrogen (nr. 2 in table 1), dissociation of hydrogen molecules (nr. 16 in table 1), and the vibrational excitation of hydrogen molecules (nr. 12 in table 1). Radiation losses occur when excited atoms decay spontaneously to a lower electronic state. The excited atoms can be produced from direct electron impact excitation (nr. 2 in table 1), the recombination of MAR dissociation product (see $\mathrm{nr} 11$ in table 1), the mutual recombination of $\mathrm{H}^{-}$(see $\mathrm{nr} 15$ in table 1), and electron-ion recombination (nr. 9 in table 1). The contribution of radiation losses for these processes are incorporated in the total energy loss of each process. The total contribution of collision processes to the plasma energy loss is shown in figure 9. Combined electronic processes such as MAR or electron-proton recombination appear to be the most dominant fraction in $Q_{\mathrm{vol}}$, with elastic collision of $\mathrm{H}^{+}-\mathrm{H}_{2}$ being the second most dominant process. The contributions of each electronic process are illustrated in figure 10 . This highlights the importance of recombination processes in removing a significant fraction of heat flux by removing plasma particles that carry and transfer their ionization potential to the target surface. However, as shown from figure 5, the contribution of plasma recombination losses are mediated by electron-proton 

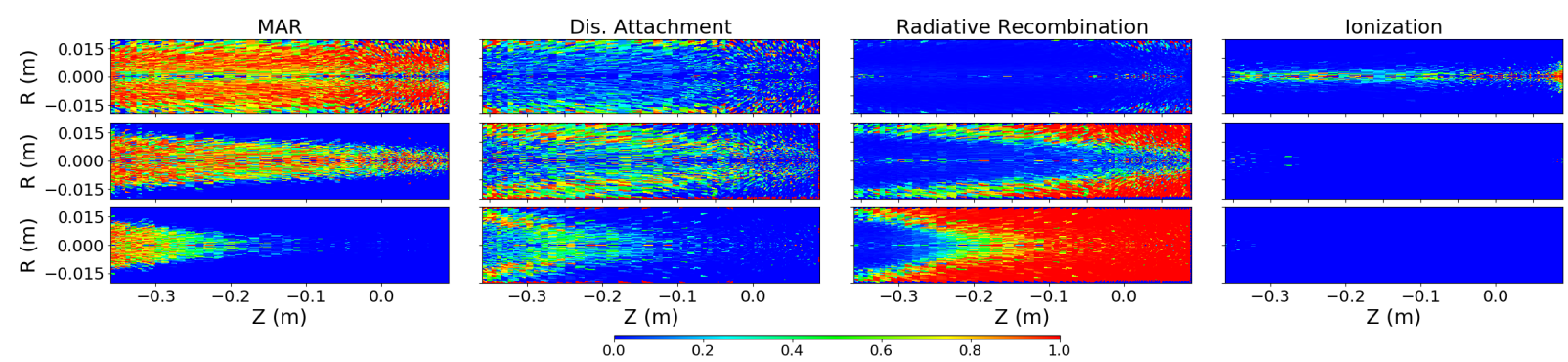

Figure 6: The spatial distribution of plasma particle losses within the plasma beam in the low density case, locally normalized to $\Phi_{\mathrm{vol}}$ in each grid cell. The particle losses are molecular activated recombination (MAR) (collision 10 and 11 in table 1), dissociative attachment (collision 14 and 15 in table 1), and electron-proton recombination (collision 9 in table 1). The distribution is shown for $p_{\mathrm{n}}=0.27 \mathrm{~Pa}$ (top row), 2.0 $\mathrm{Pa}$ (middle row) and 4.4 $\mathrm{Pa}$ (bottom row).

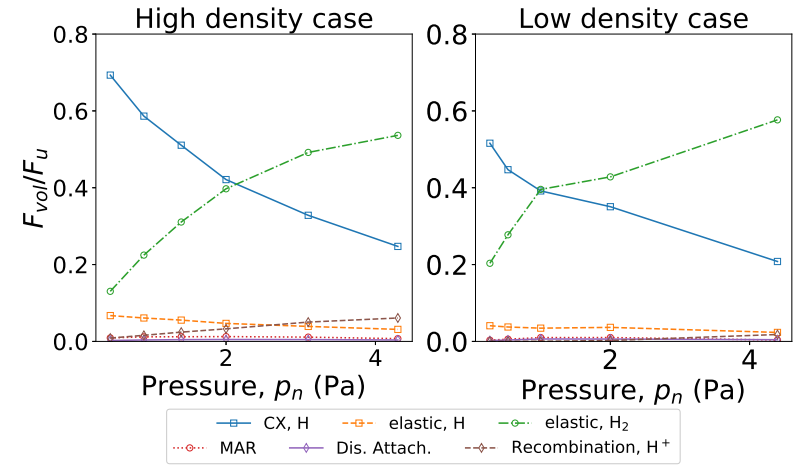

Figure 7: Individual contributions of different plasma collision processes to the volumetric momentum loss, $F_{\text {vol }}$, normalized to $F_{\mathrm{u}}+F_{p}$. Both CX and elastic $\mathrm{H}^{+}$ $+\mathrm{H}_{2}$ collision dominates the momentum loss process. There is a transition of relevance from CX to elastic $\mathrm{H}^{+}-\mathrm{H}_{2}$ collision at high $p_{\mathrm{n}}$ values.

recombination with increasing $p_{\mathrm{n}}$, and this process is effective at low $T_{\mathrm{e}}$ (as shown in Appendix A). Thus, processes that remove the plasma heat and lower the plasma temperature are equally crucial in the fraction of $Q_{\mathrm{vol}}$. To demonstrate this more clearly, the contributions of different plasma collision processes are plotted in figure 11 without accounting for the corresponding $E_{\alpha}$ gains or losses (i.e. using (18) instead of (19)). Elastic collision of $\mathrm{H}^{+}-\mathrm{H}_{2}$ is the dominant mechanism at high neutral pressures for both the high and low density case. This process is shown to have increasing relevance with increasing $p_{\mathrm{n}}$, which is expected considering that $p_{\mathrm{n}}$ is increased by injection of $\mathrm{H}_{2}$ gas into the chamber, and so increases $\mathrm{H}_{2}$ density (see Appendix B). It appears that this process occurs primarily within the bulk of the plasma, as shown in figure 12 for the low density case. At low $p_{\mathrm{n}}$, this process occurs predominantly on the plasma periphery, and as the gas pressure is increased it becomes more dominant inside the beam. At high electron temperature, which is indicated by the lowest $p_{\mathrm{n}}$ value in the low density case, CX and electronic processes are mainly responsible for heat loss in the center of the plasma beam. It should be highlighted that the dominant role of CX in the center of the beam is also due to the existence of atomic $\mathrm{H}$ sources, mainly through the dissociation of $\mathrm{H}_{2}$ as shown in figure 14.

Without taking into account the energy loss fraction by ionization potential loss, the electronic processes are only relevant at high $T_{\mathrm{e}}$ in removing the plasma heat. It even resulted in plasma heat gain for the high density case, particularly at very high $p_{\mathrm{n}}$. This is caused by electron-proton recombination as shown in figure 13. The electron-proton recombination process heats the electrons by three-body recombination, in which the secondary electron takes away the ionization potential energy 29]. In the high density case, where $n_{\mathrm{e}}$ is sufficiently high, the heating effect of threebody recombination is larger than radiative cooling, thereby constitutes a net electron heating. For MAR, the electron energy loss term corresponds to the loss of electron energy during the recombination of the hydrogenic ion $\mathrm{H}_{2}^{+}$, where the energy is transferred to the dissociation products. As shown in figure 13 . the contribution of MAR in reducing $T_{\mathrm{e}}$ does not scale with $p_{\mathrm{n}}$. Therefore, in Magnum-PSI, plasma cooling is enhanced by the increased of neutral pressure primarily through ion-molecule elastic collisions.

One can see from figure 13 that there exists a net electron energy loss through the vibrational excitation of $\mathrm{H}_{2}$ molecules to a higher state. In the simulations presented in this paper, this is accommodated with the transport of vibrationally excited $\mathrm{H}_{2}$ molecules from the plasma into the gas bulk and eventually to the cold chamber walls, where the molecules are de-excited into the ground state. Other de-excitation pathways are radiative decay and relaxation of the vibrationally 

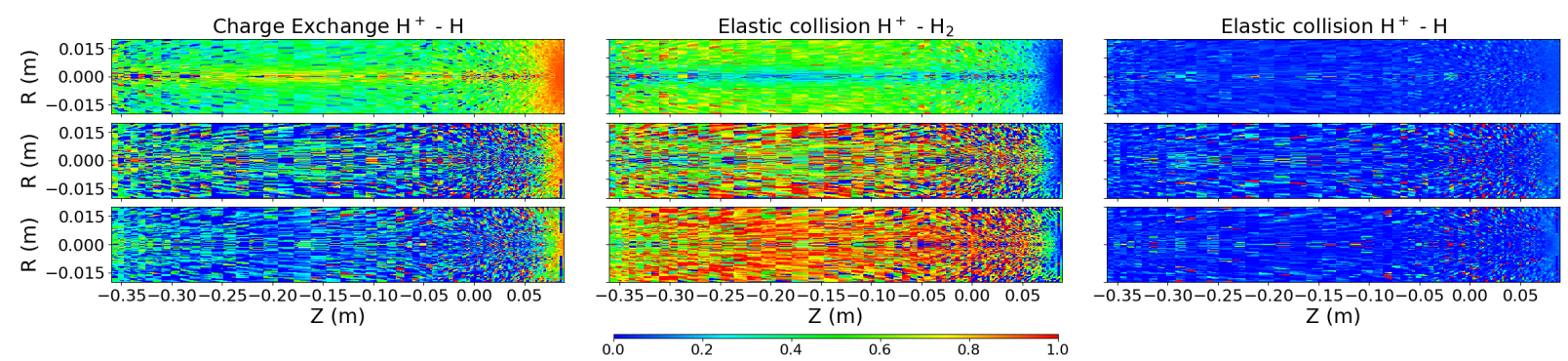

Figure 8: The spatial distribution of plasma momentum losses within the plasma beam in the low density case, locally normalized to $F_{\text {vol }}$ in each grid cells. The largest momentum losses shown here are due to charge exchange, elastic collisions of $\mathrm{H}_{2}-\mathrm{H}^{+}$, and elastic collisions of $\mathrm{H}-\mathrm{H}^{+}$. The distribution is shown for $p_{\mathrm{n}}=0.27 \mathrm{~Pa}$ (top row), 2.0 $\mathrm{Pa}$ (middle row) and 4.4 $\mathrm{Pa}$ (bottom row).

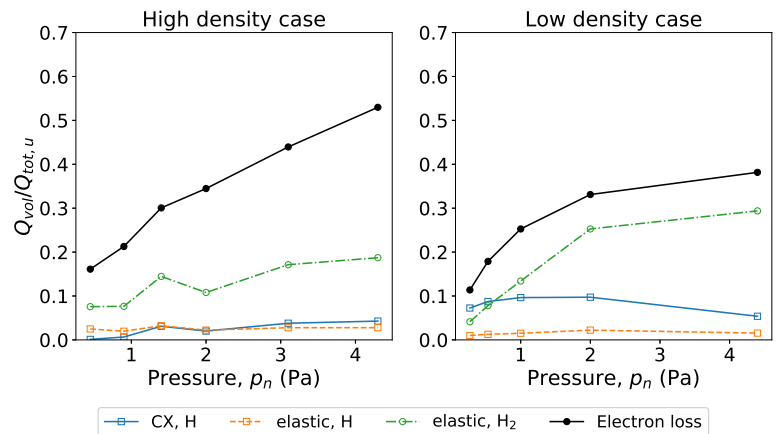

Figure 9: Individual contributions of different plasma collision processes to the volumetric energy loss, $Q_{\mathrm{vol}}$, normalized to $Q_{\mathrm{u}}$. The electron loss is the aggregate of all inelastic collision involving electrons, such as MAR or dissociation of $\mathrm{H}_{2}$. The energy loss due to electronic processes are the dominant mechanism in reducing heat flux to the target, with elastic collisions of $\mathrm{H}_{2}-\mathrm{H}^{+}$being the second most dominant process.

excited molecules with the gas. The former is not expected to be significant in the plasma regime discussed in this paper, and the latter is not expected to be significant as the gas density $\left(\mathrm{H}_{2}\right.$ and $\left.\mathrm{H}\right)$ is low within the plasma beam. When the excited molecules undergo electronic de-excitation within the plasma, the excitation energy is returned to the electrons. Thus, the net electron energy loss is the difference between the integrated electron energy loss from excitation and energy gain from de-excitation. The transport seems to be concentrated at the periphery of the plasma beam in the low density case as shown in figure 14. When $p_{\mathrm{n}}$ is increased, this process becomes the dominant electron energy loss channel, albeit relatively small compared to the total plasma heat loss at $\sim 10 \%$. As previously mentioned, the source of atomic $\mathrm{H}$ that contributes to $\mathrm{CX}$ is produced by the dissociation of $\mathrm{H}_{2}$, which is

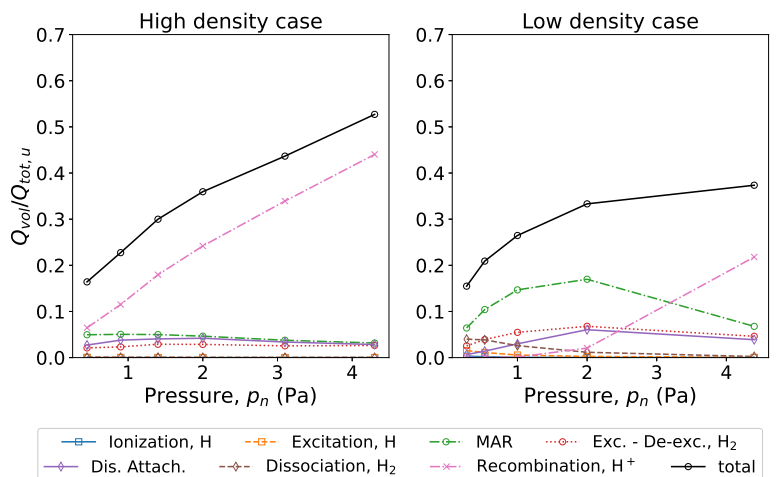

Figure 10: Individual contributions of different electronic processes corresponding to the electron loss term shown in figure 9. The different contributions are normalized to $Q_{\mathrm{u}}$. In the high density case, electronproton recombination is the dominant electron cooling term at high $p_{\mathrm{n}}$, while in the low density case it is only significant at the highest $p_{\mathrm{n}}$ value. At lower $p_{\mathrm{n}}$ MAR is the dominant process.

clearly demonstrated to be concentrated at the beam center. Another process that results in electron energy loss is the excitation of $\mathrm{H}$ atoms and the spontaneous emission of photons to the ground state. This process is shown to be localized near the target where the density of $\mathrm{H}$ is high and at the beam center. Both dissociation of $\mathrm{H}_{2}$ and excitation of $\mathrm{H}$ are relatively important only at the low density case with $p_{\mathrm{n}}=0.27 \mathrm{~Pa}$, where $T_{\mathrm{e}} \sim 3$ $\mathrm{eV}$.

\section{Effect of vibrationally excited molecules on collision rates}

B2.5-Eunomia treats vibrationally excited $\mathrm{H}_{2}$ molecules as test particles. The density, temperature, and flow velocity are resolved for states $v=0-14$. It is known that vibrationally excited molecules increase the effec- 


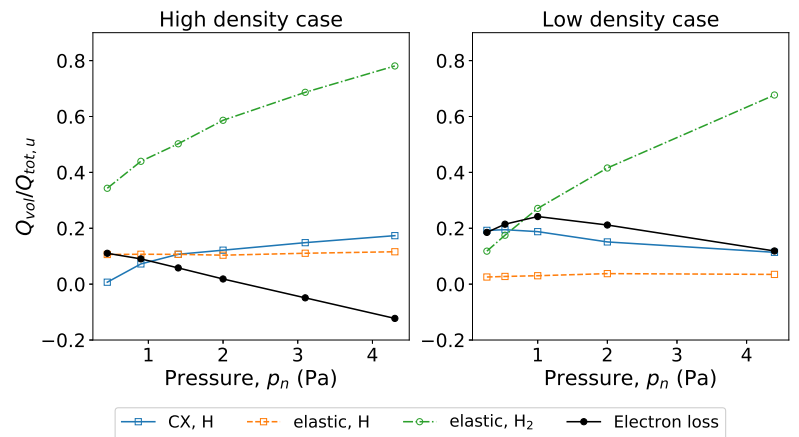

Figure 11: Individual contributions of different plasma collision processes to the volumetric heat loss, $Q_{\mathrm{vol}}$, normalized to $Q_{\mathrm{u}}$, where the contribution of ionization potential $\Phi_{\mathrm{vol}} E_{\alpha}$ and $\Phi_{\mathrm{u}} E_{\alpha}$ are omitted. Elastic collisions of $\mathrm{H}_{2}-\mathrm{H}^{+}$are the most dominant plasma heat loss channel at high neutral pressure, $p_{\mathrm{n}}$.

tive rate of MAR and dissociative attachment (collision number 10 and 14 in table 1) 30]. When these molecules are not treated as test-particles, the population distribution is assumed to be in equilibrium for a given electron temperature using a quasi-steady state (QSS) model. This particular approach neglects the separate effects of vibrational molecule transport and is likely to give an overestimate of the recombination rates 31. In a B2-EIRENE study of detachment in ASDEX-U the detached plasma solution is found to reattached when the vibrationally excited $\mathrm{H}_{2}$ molecules are treated as test particles 32. Here the rates of MAR and dissociative attachment will be compared between the QSS model and vibrationally excited densities resolved by B2.5-Eunomia. The QSS model employed in this paper resolves the density fractions of vibrationally excited $\mathrm{H}_{2}$ using the collision rates listed in table 1 In steady state, the density of $\mathrm{H}_{2}(v), n_{v}^{\mathrm{H}_{2}}$, can be expressed in linear form:

$\left(C_{(v, v+1)}+C_{(v, v-1)}+D_{v}\right) n_{v}^{\mathrm{H}_{2}}=$

$n_{v-1}^{\mathrm{H}_{2}} C_{(v-1, v)}+n_{v+1}^{\mathrm{H}_{2}} C_{(v+1, v)}$

where $v$ is the vibrational level ranging from $v=$ $0-14, C_{(v, v+1)}$ and $C_{(v, v-1)}$ are the rate coefficients of, respectively, excitation and de-excitation of the vibrational state level $v$ via electron impact and $D_{v}$ is the rate coefficient of dissociation of the vibrational molecule. The dissociation of $\mathrm{H}_{2}(v)$ occurs in three different processes, namely MAR, dissociative attachment, and electron impact dissociation (collision number 10, 14 and 16 from table 1). Therefore, the rate coefficient $D_{v}$ is the sum of the three processes and can be expressed as:

$D_{v}=D_{v, \text { MAR }}+D_{v, \text { Dis.Attach. }}+D_{v, \text { Dissociation }}$
The rate coefficients $C$ and $D$ are $T_{\mathrm{e}}$ dependent rates from the H2VIBR database 24. Using the $n_{\mathrm{e}}$ and $T_{\mathrm{e}}$ values gained from B2.5-Eunomia, the equilibrium distributions of $\mathrm{H}_{2}(v)$ in radial and axial positions can be obtained by solving the coupled linear equations of (20). The equilibrium distributions are then used to obtain the total collision rate for processes involving $\mathrm{H}_{2}(v)$ that result in plasma recombination i.e. MAR and dissociative attachment (collision number 10 and 14 from table 11). The equilibrium distribution is also resolved in B2.5-Eunomia with the addition of particle transport. The total collision rate of MAR and dissociative attachment using the density distribution resolved by B2.5-Eunomia is compared with the QSS model and shown in figure 15 . It can be observed that both MAR and dissociative attachment rates are overestimated by more than a factor of 2 on average when the transport of vibrational species is neglected. This overestimation is larger near the plasma beam center and results in a very peaked collision rate with QSS compared to the flat profile obtained using the B2.5-Eunomia distribution. The collision rates of MAR and dissociative attachment are greatly enhanced by the presence of highly vibrationally excited states $\mathrm{H}_{2}(v=p, p \geq 4)$ 24. Thus, the distribution of these highly excited states has a great influence in determining the collision rates and consequently, plasma recombination. To elucidate this further, the population density fraction of $\mathrm{H}_{2}(v=p, p \geq 4)$ is summed and compared between the QSS model and B2.5-Eunomia. The ratio of the population between the QSS model and B2.5Eunomia is shown in figure 16 . At $Z=0$ the population fraction of highly excited vibrational $\mathrm{H}_{2}$ molecules are clearly overpopulated when the transport of these molecules is neglected. In addition, the profile indicates a peaked ratio at the center of the beam, which is in line with the QSS approximation of MAR rates in figure 15. A recent Magnum-PSI experiment with similar plasma parameters, but higher molecular density, used optical emission spectroscopy (OES) to measure the population density of excited atomic $\mathrm{H}$ and found a hollow density profile 33. The hollow profile was attributed to the deficiency of MAR in the beam center which is caused by the rarefaction of molecular $\mathrm{H}_{2}$. While the rarefaction is also observed in the B2.5-Eunomia simulation of this paper (see Appendix B, using the QSS model with the same rarefied molecular density profile does not yield a hollow rate profile as seen in figure 15. It is rather caused by the transport of $\mathrm{H}_{2}(v)$. However, further investigation is required as the transport of $\mathrm{H}_{2}$ in this simulation does not produce a very pronounced hollow profile as was observed in the OES experiment. It is observed that the role of $\mathrm{H}_{2}(v)$ transport in reducing 

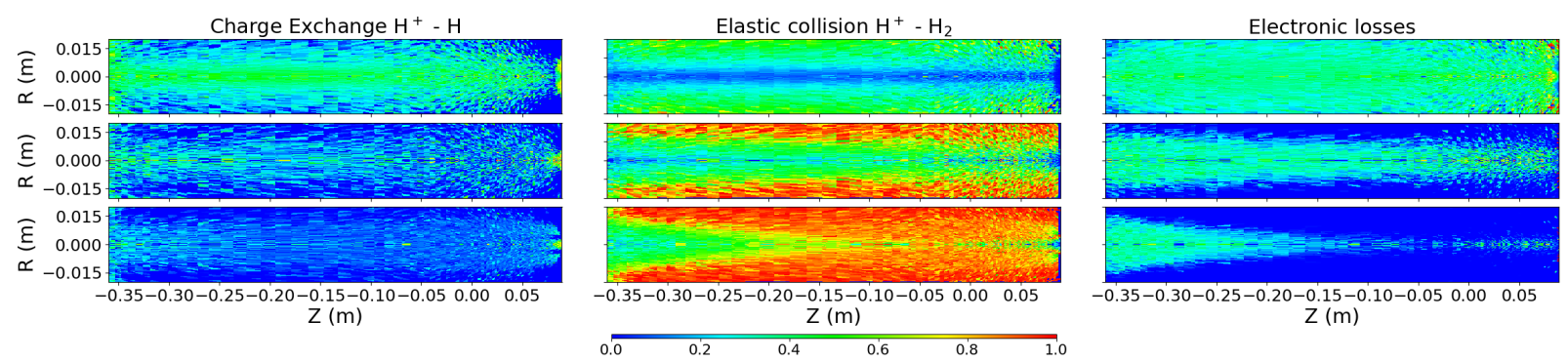

Figure 12: The spatial distribution of plasma heat losses within the plasma beam in the low density case, locally normalized to $Q_{\mathrm{vol}}$ in each grid cells. The heat losses are from charge exchange, $\mathrm{H}^{+}-\mathrm{H}_{2}$ elastic collision, and the aggregate of electron energy losses. The distribution is shown for $p_{\mathrm{n}}=0.27 \mathrm{~Pa}$ (top row), $2.0 \mathrm{~Pa}$ (middle row) and 4.4 $\mathrm{Pa}$ (bottom row).

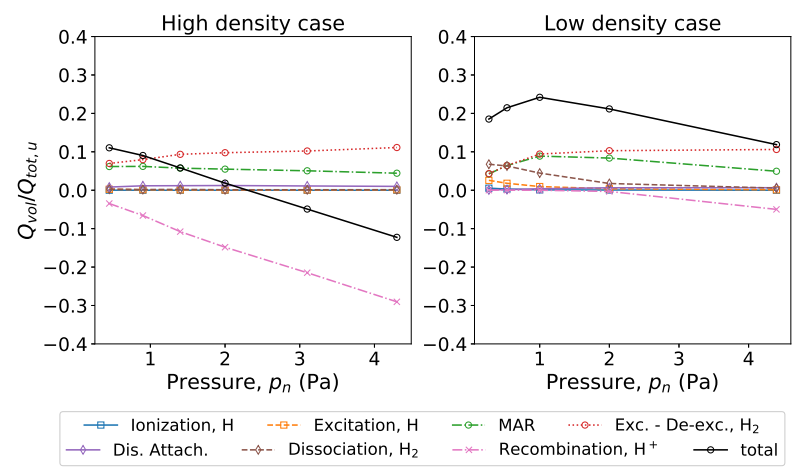

Figure 13: Individual contributions of different electronic processes corresponding to the electron loss term shown in figure 11. Electron-ion recombination yields plasma heating for the high density case and at high $p_{\mathrm{n}}$ for the low density case. MAR has significant contributions in the electron loss term, but it does not scale with $p_{\mathrm{n}}$. There is an additional electron loss term due to the vibrational excitation of molecules and its subsequent transport exiting the plasma beam.

the effective MAR rate in the center of the beam is less pronounced near the target chamber entrance, as indicated by the population ratio at $\mathrm{Z}=-0.32 \mathrm{~m}$, shown in figure 16. At this location the population of the higher vibrational states is better captured by the QSS model, with the effects of transport more prominent off axis. Moreover, the MAR rate is more relevant in this location compared to $\mathrm{Z}=0$ as shown in figure 6 . Thus, the overestimation of the MAR rate due to the neglect of high states $\mathrm{H}_{2}(v)$ transport is somewhat lessened as the largest impact is not where the MAR rate has the most significant contribution. In addition, a change in the MAR rate may not significantly change the relevant quantities in detachment, namely the particle and heat flux, due to the interchange with 3-body recombination, as was shown in a SOLPS study 34 .
The mechanism behind this interchange can be nonexistent or achieved differently in a linear device. This can be investigated with a comparison of the simulation using QSS rates and the result of this paper in future studies. Regardless of the effect to detachment characteristics, transport remains important when a local analysis of the MAR rate is done to compare, for example, with experimental measurements.

\section{Collision processes in different plasma regimes}

The B2.5-Eunomia simulations presented in this paper provide information regarding the plasma parameters $T_{\mathrm{e}}$ and $n_{\mathrm{e}}$ ranging from, respectively, $0.1-4.0 \mathrm{eV}$ and $10^{19}-10^{20} \mathrm{~m}^{-3}$, distributed in radial and axial positions. The particle and energy losses for the atomic and molecular processes listed in table 1 and described extensively in section 4 are also distributed in radial and axial positions. A general insight can be obtained by mapping the unique collisions to their local $T_{\mathrm{e}}$ and $n_{\mathrm{e}}$ values. The mapping of collisions responsible for plasma particle sources and sinks are shown in figure 17. The placement of these processes in $T_{\mathrm{e}}$ and $n_{\mathrm{e}}$ space are determined using weighted averaging and the weighting value is the collision rate normalized to $n_{\mathrm{e}}$ (which becomes $\langle\sigma v\rangle n$ ). The ionization of atomic $\mathrm{H}$ is the only process responsible for plasma particle source and occur at the highest $T_{\mathrm{e}}$ in the parameter space. MAR is the most effective at $T_{\mathrm{e}}=1.5 \mathrm{eV}$, with dissociative attachment being effective at slightly lower $T_{\mathrm{e}}=0.8 \mathrm{eV}$. As expected, atomic recombination (radiative and three-body) is effective primarily at the lowest $T_{\mathrm{e}}$ in the parameter space, in this case $T_{\mathrm{e}}=0.3 \mathrm{eV}$. The error bars in figure 17 indicate the standard deviation of the weighted average. There is a clear separation between ionization and recombination processes at around $T_{\mathrm{e}}=2 \mathrm{eV}$. There is also a clear separation between MAR and atomic recombination, with dissociative attachment acting as a bridging gap 

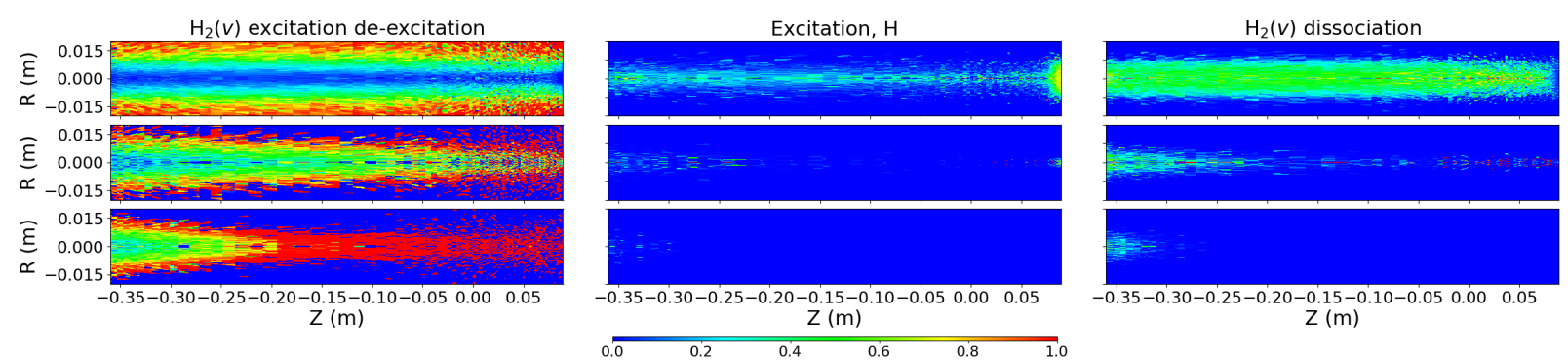

Figure 14: The spatial distribution of electron energy losses within the plasma beam in the low density case, locally normalized to the total electron energy loss in each grid cells. The processes listed here are the net loss from vibrational excitation and de-excitation of $\mathrm{H}_{2}$, excitation of $\mathrm{H}$, and the dissociation of $\mathrm{H}_{2}$. The distribution is shown for $p_{\mathrm{n}}=0.27 \mathrm{~Pa}$ (top row), 2.0 $\mathrm{Pa}$ (middle row) and $4.4 \mathrm{~Pa}$ (bottom row).
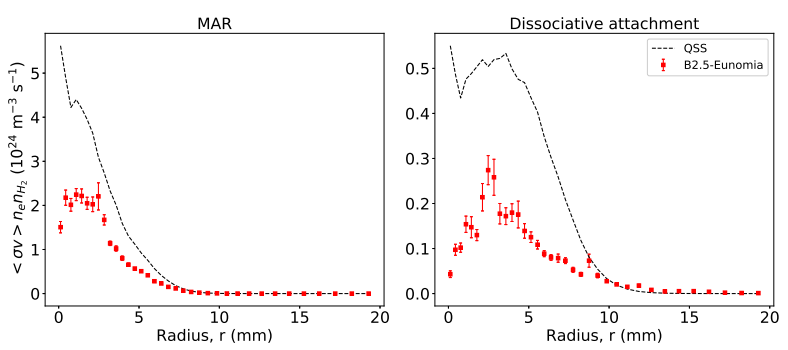

Figure 15: The collision rate profile for MAR and dissociative attachment processes at the target TS position $(\mathrm{Z}=0)$, using the plasma and $\mathrm{H}_{2}$ densities of the low density case where $p_{\mathrm{n}}=2 \mathrm{~Pa}$. The collision rates are evaluated using the equilibrium distribution of vibrationally excited $\mathrm{H}_{2}$ from the QSS model and simulation results. The error bars on B2.5-Eunomia results are the standard error of the $\mathrm{H}_{2}$ density caused by statistical noise. The QSS rate overestimates MAR and dissociative attachment by more than a factor of 2 on average.

between the two processes. This separation was also illustrated in figure 6. Recent experiments in TCV investigated the role of plasma-molecule interaction using measurements of Balmer emission and observed the increase of MAR rate during detachment, with the MAR rate being larger than electron-ion recombination at electron temperatures around $1.5-3 \mathrm{eV}$ [35]. It was also suggested that near the divertor target where the temperature is about $1-3 \mathrm{eV}, \mathrm{MAR}$ is accommodated by vibrationally excited molecules, which are simulated in this paper. This observation is in line with the separation of relevancy between MAR and atomic recombination shown in figure 6 .

It was made clear in section 4.3 that plasma recombination removes a significant part of heat flux going into the target by removing plasma particles that would deposit their ionization potential. Atomic recombination, which is the most important process to

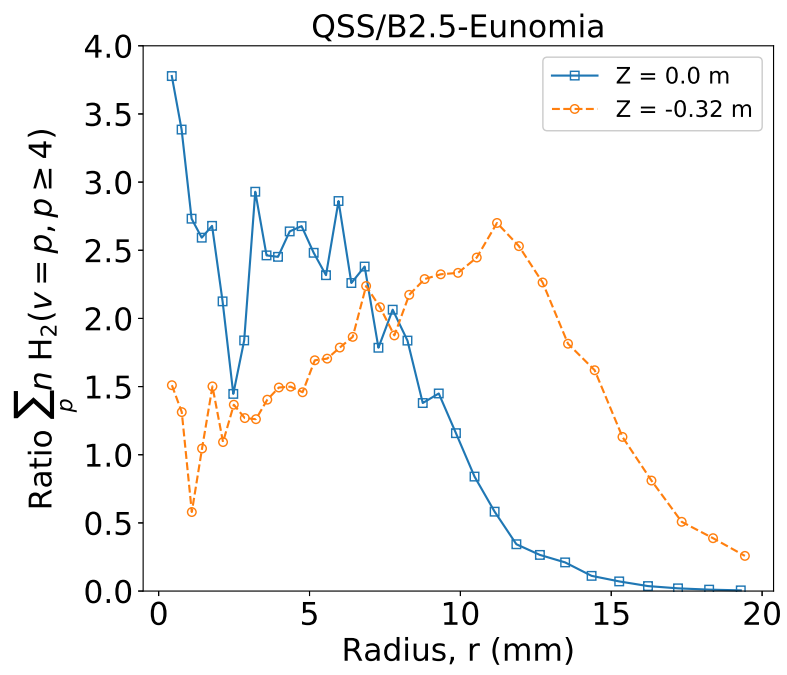

Figure 16: The ratio of population density fraction between the QSS model and B2.5-Eunomia. The population density is the sum of all vibrationally excited $\mathrm{H}_{2}(v)$ where $v=4-14$. Values greater than 1 indicate an overpopulation of these states by the QSS model. The population ratio at $\mathrm{Z}=0$ shows a peaked overpopulation profile by the QSS model suggesting transport of the highly vibrational states in the beam center. By contrast, near the target chamber entrance at $\mathrm{Z}=-0.32 \mathrm{~m}$, the overpopulation profile is off-center.

achieve total detachment, is only effective in very low $T_{\mathrm{e}}$, and other processes are responsible for lowering this temperature. These processes can be mapped similarly to figure 17 with the weighting value changed from collision rate to heat loss rate normalized to $n_{\mathrm{e}}$ (or $Q_{\mathrm{vol}} / n_{\mathrm{e}}$ ). The mapping is shown in figure 18 . The excitation of atomic $\mathrm{H}$ and dissociation of $\mathrm{H}_{2}$ are relevant primarily in high $T_{\mathrm{e}}$ at around $T_{\mathrm{e}}=2.5$ $\mathrm{eV}$ similarly to ionization of atomic $\mathrm{H}$. The electron energy loss due to transport of vibrationally excited $\mathrm{H}_{2}$ molecules (H2 exc. de-exc. in figure 18) is centered 


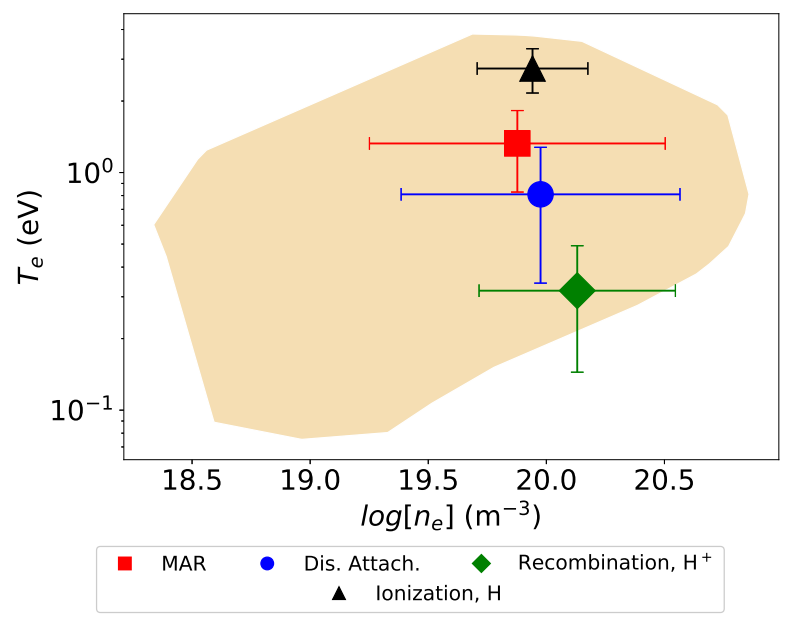

Figure 17: The distribution of processes contributing to plasma particle sources and sinks in plasma parameter space, $T_{\mathrm{e}}$ and $n_{\mathrm{e}}$. The location is based on the average location of each process weighted on their respective collision rates normalized to $n_{\mathrm{e}}$. The error bars indicate the standard deviation of the average. The colored patch represents the span of $T_{\mathrm{e}}$ and $n_{\mathrm{e}}$ values from simulations of the high and low density case including all neutral pressures.

around $T_{\mathrm{e}}=1 \mathrm{eV}$. For CX and elastic collisions, which are elastic $\mathrm{H}^{+}-\mathrm{H}$ and elastic $\mathrm{H}^{+}-\mathrm{H}_{2}$, the variance of the mappings is rather large. Elastic $\mathrm{H}^{+}-\mathrm{H}_{2}$ collisions are the most dominant heat loss channel and play a significant role in the low $T_{\mathrm{e}}$ regime, where $T_{\mathrm{e}} \leq 1$ $\mathrm{eV}$. This is reflected in figure 12 where these collisions are relatively more relevant in the plasma beam edges compared to the beam center. The reasoning would be that at higher $T_{\mathrm{e}}, \mathrm{H}_{2}$ dissociation or molecular recombination processes remove the molecules from participating in the heat exchange. The resulting atomic product however, can undergo elastic collision or CX, and so these collisions are centered in a higher $T_{\mathrm{e}}$ than elastic $\mathrm{H}^{+}-\mathrm{H}_{2}$ collision as seen in figure 18 . Elastic $\mathrm{H}^{+}-\mathrm{H}_{2}$ collisions as a power loss channel at low electron temperatures are also found in several tokamak studies $[5,10,36$.

\section{Conclusion and outlook}

This paper is the second part of a detailed study of the detachment experiments in Magnum-PSI. The plasma volumetric losses i.e., particle, momentum and energy losses have been analyzed in detail. The volumetric particle and energy losses appear to scale almost linearly with the neutral background pressure which is increased by gas puffing. The volumetric

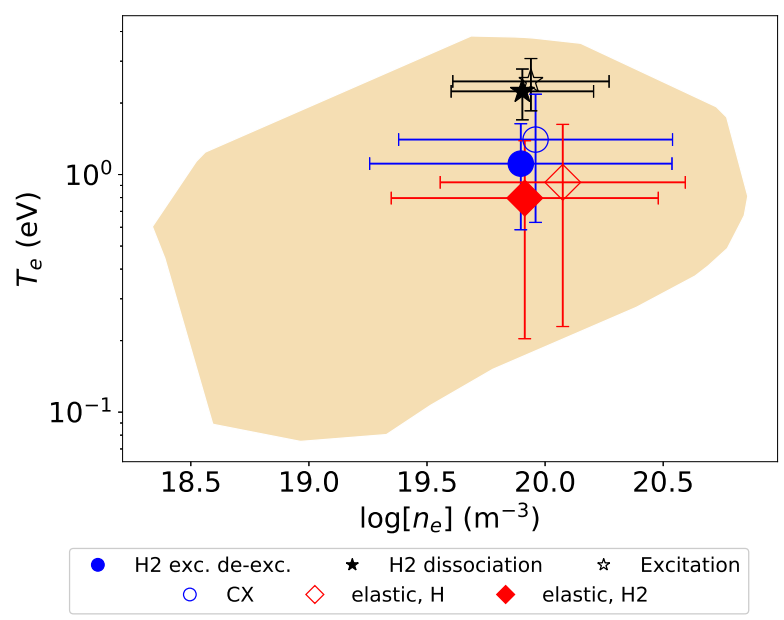

Figure 18: The distribution of processes contributing to plasma heat loss in plasma parameter space, $T_{\mathrm{e}}$ and $n_{\mathrm{e}}$. The location is based on the average location of each process weighted on their respective amount of heat loss normalized to $n_{\mathrm{e}}$. The error bars indicate the standard deviation of the average. The colored patch represents the span of $T_{\mathrm{e}}$ and $n_{\mathrm{e}}$ values from simulations of the high and low density case including all neutral pressures.

momentum loss does not appear to linearly scale with the increase of neutral background pressure, due to the interchange between $\mathrm{CX}$ and elastic $\mathrm{H}^{+}-\mathrm{H}_{2}$ collision (respectively, collision 3 and 8 in table 1) contributions to the momentum loss. The particle losses are primarly caused by MAR (nr. 10 and 11 in table 11) in the temperature range of $T_{\mathrm{e}} \approx 1.5$ $\mathrm{eV}$, with atomic recombination (nr. 9 in table 1) being dominant in the very low temperatures $T_{\mathrm{e}} \leq$ $1 \mathrm{eV}$. Dissociative attachment (nr. 14 and 15 in table 1) never emerges as the dominant process in all plasma parameters discussed in this paper. Plasma momentum loss are primarily caused by $\mathrm{CX}$ and elastic $\mathrm{H}^{+}-\mathrm{H}_{2}$ collisions. Momentum loss by CX occurs near the target where the density of $\mathrm{H}$ is high due to surface recombination and the beam center where $\mathrm{H}$ is produced via dissociation of $\mathrm{H}_{2}$, and momentum loss by elastic $\mathrm{H}^{+}-\mathrm{H}_{2}$ collisions occur in the plasma bulk. The main contributor to target heat flux reduction is plasma recombination (MAR, dissociative attachment and atomic recombination) by reducing the particle flux to the target and thereby reducing the deposited ionization potential energy. Before the most relevant atomic recombination can occur effectively, the plasma is cooled primarily by elastic $\mathrm{H}^{+}-\mathrm{H}_{2}$ collision. There are electron cooling channels due to dissociation of $\mathrm{H}_{2}$ at higher $T_{\mathrm{e}}$ and transport of vibrational $\mathrm{H}_{2}$. However, 
their contributions to the overall power balance are relatively small and does not scale with increasing neutral background pressure.

The transport of highly vibrational states of $\mathrm{H}_{2}$ is important to include when determining the recombination rate due to MAR or dissociative attachment. Without transport, the MAR rate can be overestimated by a factor of 2-3. The highest impact of transport on MAR occurs at temperatures where MAR is not the most significant contributor to plasma particle losses, and so the impact of transport on the global MAR rate is reduced. However, the impact of transport on the MAR rate should be considered when calculating the local MAR rate. Each collisional process is mapped in the plasma parameter space, $T_{\mathrm{e}}$ and $n_{\mathrm{e}}$, in order to define their relevance in different plasma regimes. Ionization of $\mathrm{H}$ (collision 1 in table 1) as a process of plasma particle source are relevant in high $T_{\mathrm{e}}$ regimes of around $T_{\mathrm{e}}=2.5$ $\mathrm{eV}$ and separate from plasma recombination processes. MAR and atomic recombination occupy separate $T_{\mathrm{e}}$ regimes, with MAR being relevant around $T_{\mathrm{e}}=1.5 \mathrm{eV}$ and atomic recombination at the low temperature of $T_{\mathrm{e}}=0.3 \mathrm{eV}$. Dissociative attachment contributes most to plasma recombination in the temperature regime between MAR and atomic recombination. Elastic $\mathrm{H}^{+}$$\mathrm{H}_{2}$ collisions are the primary plasma cooling channel and occur effectively in the low temperature regions where $T_{\mathrm{e}} \leq 1 \mathrm{eV}$, with $\mathrm{CX}$ being effective around $T_{\mathrm{e}}=1.5 \mathrm{eV}$. The plasma cooling due to transport of vibrationally excited $\mathrm{H}_{2}$ is effective at around $T_{\mathrm{e}}=1 \mathrm{eV}$. At high temperatures where $T_{\mathrm{e}} \geq 2 \mathrm{eV}$, electronic cooling due to excitation of atomic $\mathrm{H}$ and $\mathrm{H}_{2}$ dissociation are relevant.

In this paper we have shown the importance of different collisional processes in a plasma undergoing detachment in Magnum-PSI and associating them with different plasma regimes. Several processes that are highlighted in this paper, namely MAR and elastic $\mathrm{H}^{+}$$\mathrm{H}_{2}$ collisions, have been found to occur in tokamak divertors undergoing detachment $5,3,10,35,36$. It is important to note that in the simulations of this paper and experiments, these processes are exclusively influenced by the increase of neutral background pressure via gas puffing of $\mathrm{H}_{2}$. Other aspects of tokamak divertor detachment are neglected, such as the effect of impurity seeding, geometrical drifts, isotope effects, and divertor closure. Divertor closure is especially an important aspect since in MagnumPSI the neutral pressure in the target chamber is well maintained by the skimmers and the additional effect of plasma-neutral drag, which can be difficult to attain in a divertor. Future comparisons between the simulation results discussed here and simulations of tokamak divertor closure can further elucidate the role of plasma-neutral interaction in reaching plasma detachment and consequently, the particle and heat flux to the divertor targets. The results can also be used to compare with future simulations which incorporate radiation trapping. Photon reabsorption is expected to lessen the electronic energy loss channel in the simulation 37]. However, if elastic $\mathrm{H}^{+}-\mathrm{H}_{2}$ collision is indeed the primary energy loss channel during Magnum-PSI detachment, the effect of radiation trapping to the overall energy balance should be minimal, since the photons are not an important loss channel in the simulation.

\section{Data availability}

The data that support the findings of this study are openly available in Zenodo at https://dx.doi.org/ 10.5281/zenodo.5116241, reference number [38].

\section{Acknowledgements}

Differ is part of the institutes organisation of NWO. This work is part of the research programme Taming the Flame, which is partly financed by NWO. This work has been carried out within the framework of the EUROfusion Consortium and has received funding from the Euratom research and training programme 2014-2018 and 2019-2020 under grant agreement No 633053. The views and opinions expressed herein do not necessarily reflect those of the European Commission. This work was carried out on the Dutch national e-infrastructure with the support of SURF Cooperative. In addition, we acknowledge computing resources provided on the Marconi-Fusion HPC infrastructure.

\section{Appendix A. Rate coefficients of relevant collisions}

The rate coefficients of relevant collisions discussed in this paper are shown in figure A1.

\section{Appendix B. Neutral densities in front of the target}

The radial density profile of neutral hydrogen and hydrogen molecules (the aggregate of all vibrational states) in front of the target is shown in figure B1. The densities are obtained from the low density case simulations for neutral background pressures, $p_{\mathrm{n}}=$ 0.27 Pa, 2.0 Pa, and 4.4 Pa.

\section{References}

[1] Loarte A, Monk R, Martín-Solís J, Campbell D, Chankin A, Clement S, Davies S, Ehrenberg J, Erents S, Guo H, 


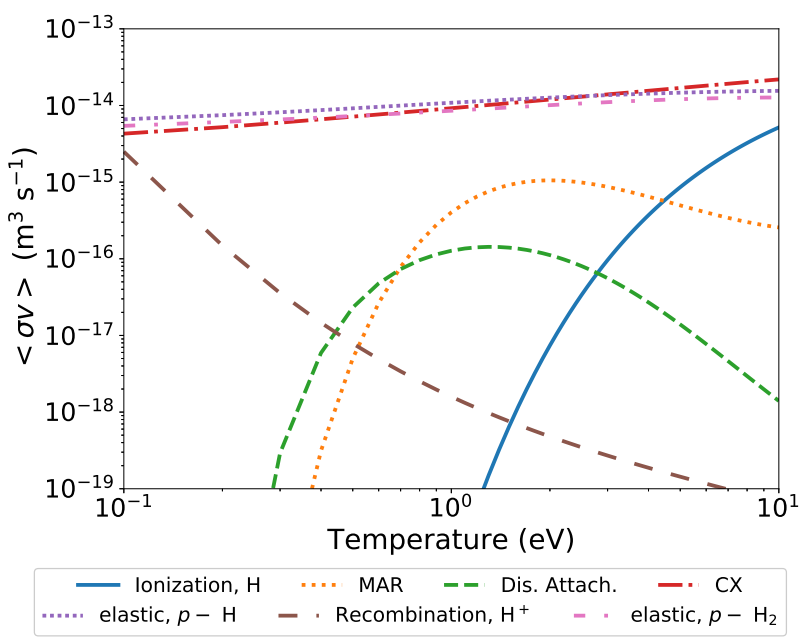

Figure A1: The rate coefficients of relevant collision rates. The ionization of $\mathrm{H}$ (collision 1 in table 1) and charge exchange (CX) (collision 3 in table 1) rate coefficients are obtained from 22 . The other rate coeffcients are obtained from 23 . The rate coefficients of MAR (nr. 10 and 11 in table 1) and dissociative attachment (nr. 14 and 15 in table1) are the effective rate coefficients assuming vibrational $\mathrm{H}_{2}$ distribution in local thermal equilibrium. The rate coefficient of $\mathrm{H}^{+}$recombination (nr. 9 in table 1 ) is temperature and density dependent. In this figure the electron density is assumed to be $10^{14} \mathrm{~cm}^{-3}$
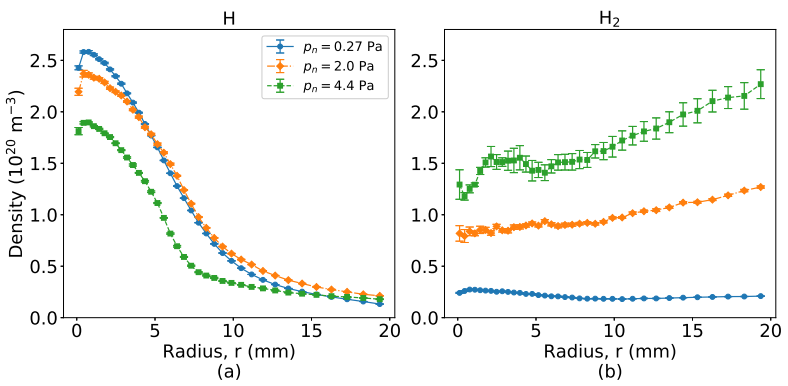

Figure B1: The radial profiles of neutral densities for (a) atomic hydrogen and (b) molecular hydrogen. The profiles are taken in front of the target at $\mathrm{Z}=0.087 \mathrm{~m}$. The error bars indicate the standard deviation of the average based on several simulation cycles.

Harbour P, Horton L, Ingesson L, Jäckel H, Lingertat J, Lowry C, Maggi C, Matthews G, McCormick K, O'Brien D, Reichle R, Saibene G, Smith R, Stamp M, Stork D and Vlases G 1998 Nuclear Fusion 38 331-371 URL https://doi.org/10.1088/0029-5515/38/3/303

[2] Krasheninnikov S I and Kukushkin A S 2017 Journal of Plasma Physics $\mathbf{8 3} 155830501$

[3] Stangeby P 2019 Plasma Physics and Controlled Fusion 62 025012 URL https://doi.org/10.1088\%2F1361-6587\% 2Fab51a9
[4] Ohno N 2017 Plasma Physics and Controlled Fusion 59 034007 URL https://doi.org/10.1088\%2F1361-6587\% 2Faa5394

[5] Kotov V and Reiter D 2009 Plasma Physics and Controlled Fusion 51115002 URL https://doi.org/10.1088/ 0741-3335/51/11/115002

[6] Reimold F, Wischmeier M, Bernert M, Potzel S, Coster D, Bonnin X, Reiter D, Meisl G, Kallenbach A, Aho-Mantila L and Stroth U 2015 Journal of Nuclear Materials 463 128-134 ISSN 0022-3115 pLASMA-SURFACE INTERACTIONS 21 URL https://www.sciencedirect.com/ science/article/pii/S002231151400960X

[7] Wensing M, Duval B, Février O, Fil A, Galassi D, Havlickova E, Perek A, Reimerdes H, Theiler C, Verhaegh K et al. 2019 Plasma Physics and Controlled Fusion 61085029

[8] Sang C, Guo H, Stangeby P, Lao L and Taylor T 2017 Nuclear Fusion $\mathbf{5 7} 056043$

[9] Moulton D, Harrison J, Lipschultz B and Coster D 2017 Plasma Physics and Controlled Fusion 59065011

[10] Park J S, Groth M, Pitts R, Bak J G, Thatipamula S, Juhn J W, Hong S H and Choe W 2018 Nuclear Fusion 58 126033

[11] Pshenov A, Kukushkin A and Krasheninnikov S $2017 \mathrm{Nu}$ clear Materials and Energy 12 948-952 ISSN 23521791 proceedings of the 22nd International Conference on Plasma Surface Interactions 2016, 22nd PSI URL https://www.sciencedirect.com/science/ article/pii/S2352179116300655

[12] Islam M, Nakashima Y, Takechi S, Tatsumi R, Hatayama $A$, Iijima $T$, Yamashita $S$, Yoshimoto $T$, Hara $T$, Ezumi N and Sakamoto M 2019 Nuclear Materials and Energy 18 182-187 ISSN 2352-1791 URL https://www.sciencedirect.com/science/article/ pii/S2352179118302205

[13] Ješko K, Marandet Y, Bufferand H, Gunn J P, van der Meiden H J and Ciraolo G 2018 Plasma Physics and Controlled Fusion 60125009 URL https://doi.org/10. 1088/1361-6587/aae80d

[14] Perillo R, Akkermans G, Classen I, Vijvers W, Chandra $\mathrm{R}$, Jesko K, Korving S, Vernimmen $\mathrm{J}$ and de Baar M 2019 Nuclear Materials and Energy 1987 - 93 ISSN 2352-1791 URL http://www.sciencedirect.com/ science/article/pii/S2352179118300838

[15] van Eck H, Akkermans G, Alonso van der Westen $\mathrm{S}$, Aussems D, van Berkel $\mathrm{M}$, Brons S, Classen $\mathrm{I}$, van der Meiden $\mathrm{H}$, Morgan $\mathrm{T}$, van de Pol $\mathrm{M}$, Scholten J, Vernimmen J, Vos E and de Baar M 2019 Fusion Engineering and Design 14226 - 32 ISSN 0920-3796 URL http://www.sciencedirect.com/ science/article/pii/S092037961930554X

[16] Schneider R, Bonnin X, Borrass K, Coster D P, Kastelewicz H, Reiter D, Rozhansky V A and Braams B J 2006 Contributions to Plasma Physics 46 3-191 (Preprint https://onlinelibrary.wiley.com/doi/pdf/10.1002/ ctpp. 200610001) URL https://onlinelibrary.wiley. com/doi/abs/10.1002/ctpp.200610001

[17] Wieggers R C, Coster D P, Groen P W C, de Blank H J and Goedheer W J 2013 J. Nucl. Mater. 438 S643 - S646

[18] Chandra R, de Blank H J, Diomede P, van Eck H J N, van der Meiden $\mathrm{H}$ J, Morgan $\mathrm{T} \mathrm{W}$, Vernimmen J W M and Westerhof E 2021 Plasma Physics and Controlled Fusion 63095006 URL/https://doi.org/10. 1088/1361-6587/ac11b6

[19] Braginskii S 1965 Reviews of Plasma Physics 1205

[20] Jesko K 2018 Studying Divertor Relevant Plasmas in Linear Devices PhD dissertation Technische Universiteit Eindhoven

[21] Perillo R, Chandra R, Akkermans G R A, Classen I G J and Korving S Q 2019 Physics of Plasmas 26102502 
(Preprint https://doi.org/10.1063/1.5120180) URL https://doi.org/10.1063/1.5120180

[22] Reiter D 2002 Forschungszentrum Juelich GmbH $\mathbf{5 2 4 2 5}$

[23] Reiter D et al. 2000 Electronic file, FZ Jülich

[24] Reiter D 2005 Forschungszentrum Juelich GmbH

[25] Perrin J, Leroy O and Bordage M 1996 Contributions to Plasma Physics 36 3-49

[26] Krasheninnikov S I, Kukushkin A S and Pshenov A A 2016 Physics of Plasmas 23055602 (Preprint https: //doi.org/10.1063/1.4948273) URL https://doi.org/ $10.1063 / 1.4948273$

[27] Kallenbach A, Bernert M, Dux R, Casali L, Eich T, Giannone L, Herrmann A, McDermott R, Mlynek A, Müller H W, Reimold F, Schweinzer J, Sertoli M, Tardini G, Treutterer W, Viezzer E, Wenninger $\mathrm{R}$ and and M W 201355124041 URL https://doi.org/10.1088/ 0741-3335/55/12/124041

[28] Wieggers R C 2012 B2.5-Eunomia simulations of Pilot-PSI PhD dissertation Technische Universiteit Eindhoven

[29] Stangeby P C 2000 The plasma boundary of magnetic fusion devices (Institute of Physics Pub. Philadelphia, Pennsylvania)

[30] Pigarov A and Krasheninnikov S 1996 Physics Letters A $\mathbf{2 2 2}$ 251-257 ISSN 0375-9601 URL https://www.sciencedirect.com/science/article/ pii/0375960196006299

[31] Baeva M, Goedheer W J and Cardozo N J L 2008 Plasma Science and Technology 10 162-169 URL https://doi. org/10.1088/1009-0630/10/2/05

[32] Fantz U, Reiter D, Heger B and Coster D 2001 Journal of Nuclear Materials 290-293 367 - 373 ISSN 0022-3115 14th Int. Conf. on Plasma-Surface Interactions in Controlled Fusion D evices URL http://www.sciencedirect.com/science/article/ pii/S0022311500006383

[33] Akkermans G R A, Classen I G J, Perillo R, van der Meiden H J, Federici F and Brezinsek S 2020 Physics of Plasmas 27102509 (Preprint https://doi.org/ 10.1063/5.0017714) URL https://doi.org/10.1063/5. 0017714

[34] Kukushkin A, Krasheninnikov S, Pshenov A and Reiter D 2017 Nuclear Materials and Energy 12 984-988 ISSN 2352-1791 proceedings of the 22nd International Conference on Plasma Surface Interactions 2016, 22nd PSI URL https://www.sciencedirect.com/science/ article/pii/S2352179116301211

[35] Verhaegh K, Lipschultz B, Harrison J, Duval B, Bowman C, Fil A, Gahle D S, Moulton D, Myatra O, Perek A, Theiler C and Wensing M $2021 \mathrm{Nu}$ clear Materials and Energy 26100922 ISSN 23521791 URL https://www.sciencedirect.com/science/ article/pii/S2352179121000181

[36] Smolders A, Wensing M, Carli S, Oliveira H D, Dekeyser W, Duval B P, Février O, Gahle D, Martinelli L, Reimerdes H, Theiler C, Verhaegh K and the TCV team 2020 62125006 URL https://doi.org/10.1088/1361-6587/ abbcc5

[37] Pshenov A, Kukushkin A, Marenkov E and Krasheninnikov S 201959106025 URL https://doi.org/10.1088/ 1741-4326/ab3144

[38] Chandra R 2021 URL https://doi.org/10.5281/zenodo. 5116241 